\title{
Automodulación de fase en redes ópticas pasivas de próxima generación, utilizando modulación por desplazamiento cuaternario de fase
}

\section{Self phase modulation in next generation passive optical networks using quaternary phase shift keying}

\author{
Auto-modulação de fase em redes ópticas passivas de próxima geração, utilizando \\ modulação por deslocamento quaternário de fase
}

\author{
Yhon Edinson Estevez-Mendoza ${ }^{1}$, Byron Medina-Delgado², Luis Leonardo Camargo-Ariza ${ }^{3}$
}

\begin{abstract}
Forma de citar: Y.E. Estevez-Mendoza, B. Medina-Delgado y L.L. Camargo-Ariza, "Automodulación de fase en redes ópticas pasivas de próxima generación, utilizando modulación por desplazamiento cuaternario de fase", Respuestas, vol. 22, no. 1, pp. 37-47, 2017.
\end{abstract}

Recibido:

Junio 30 de 2016

Aceptado:

Noviembre 15

de 2016

'Ingeniero Electrónico yhonedinsonem@ufps.edu.co Orcid: 0000-0002-1383-2063 Universidad Francisco de Paula

Santander

Cúcuta-Colombia

${ }^{2}$ Magíster en Ingeniería

Electrónica

byronmedina@ufps.edu.co

Orcid: 0000-0003-0754-8629

Universidad Francisco de Paula

Santander

Cúcuta-Colombia

${ }^{3}$ Magíster en Ingeniería

Electrónica

lcamargoa@unimagdalena.

edu.co

Orcid: 0000-0002-7956-441X

Universidad del Magdalena Santa Marta-Colombia

\section{Resumen}

Antecedentes: la información en un canal de fibra óptica sufre distorsiones debido a la aparición de efectos lineales y no lineales, restringiendo la velocidad de transmisión. En este documento se analiza el error no lineal denominado automodulación de fase (SPM), el cual genera un desfase en los pulsos trasmitidos, ocasionando errores de bits en la comunicación. Objetivo: el proyecto está orientado a evaluar el comportamiento del error no lineal SPM en una comunicación monocanal de fibra óptica utilizando Matlab. Métodos: su desarrollo parte del modelado matemático del error SPM, para ser codificado en Matlab y evaluar su desempeño en el canal para condiciones específicas. Para realizar las simulaciones se implementó un canal de comunicaciones en fibra óptica, teniendo en cuenta los parámetros que rigen las redes XGPON o 10GPON que son la siguiente generación de Red Óptica Pasiva con Capacidad de Gigabit (GPON). Para simular el canal de fibra óptica se definieron las distancias de 20, 40 y $60 \mathrm{~km}$, y las potencias de 4, 10 y $20 \mathrm{~mW}$, con velocidades de transmisión de $10 \mathrm{Gbps}$, en las longitudes de onda de 1550 y $1310 \mathrm{~nm}$, usando la modulación QPSK. Los parámetros para desarrollar la simulación consideraron las recomendaciones de la Unión Internacional de Telecomunicaciones (ITU). Resultados: mediante un análisis gráfico se identificaron los parámetros que afectan el error SPM, como la potencia, el área efectiva, la distancia y el tipo de fibra, entre otros. Conclusión: el error SPM por sí solo no es perjudicial para las redes XGPON en la modulación QPSK, considerando que el máximo desfase obtenido en el proyecto fue de $28.8^{\circ}$, siempre y cuando se tengan en cuenta la potencia, la distancia y los tipos de fibra, de acuerdo con las recomendaciones de la ITU (G652, G987, G691 y G957).

Palabras clave: Automodulación de fase, fibra óptica monomodo estándar, modulación por desplazamiento cuaternario de fase, red óptica pasiva con capacidad de gigabit.
Abstract
Background: the information transmitted by optical fiber channels is distorted due to the appearance of linear and nonlinear effects, which restrict the transmission rate. This 
No. 1

Enero - Junio 2017 ISSN 0122-820X E-ISSN 2422-5053 PP: $37-47$

paper analyzes the nonlinear error self-phase modulation (SPM) Objective: the project aims to evaluate the behavior of nonlinear error SPM in a single channel fiber optic communication using Matlab. Methods: The mathematical modeling of error SPM was developed in order to be coded in Matlab and evaluate their performance in the channel for specific conditions. To perform the simulations a communications channel was implemented in fiber optics, taking into account the parameters governing XGPON or 10GPON networks or which are the next generation of Gigabit Passive Optical Network (GPON). In order to simulate the optical fiber channel three distances 20,40 and $60 \mathrm{~km}$, and three power values of 4,10 and $20 \mathrm{~mW}$ were defined, with transmission speeds of 10 Gbps wavelengths 1550 and $1310 \mathrm{~nm}$, respectively; using QPSK modulation. The parameters to simulate were defined following the recommendations of the International Telecommunication Union (ITU). Results: using a graphical analysis we identified the parameters that affect the error SPM, such as power, the effective area, the distance, the fiber type, among others. Conclusion: the error SPM alone is not harmful to the GPON networks in QPSK modulation, considering that the maximum phase shift obtained in the project was $28.8^{\circ}$, as far as the power, the distance and the types of fiber are taken into account according to the recommendations of the ITU (G652, G987, G691 y G957).

Keywords: Gigabit passive optical network, quaternary phase shift keying, self phase modulation, standar singlemode fiber.

\section{Resumo}

Antecedentes: ainformação num canal de fibra óptica sofre distorções devido à aparição de efeitos lineares e não lineares, restringindo a velocidade de transmissão. Neste documento analisou-se o erro não linear denominado auto-modulação de fase -Self-phasemodulation(SPM), o qual gera uma lacuna nos pulsos transmitidos, ocasionando erros de bitsna comunicação.Objetivo: o projeto esteve orientado a avaliar o comportamento do erro não linear SPM em uma comunicação de fibra óptica de canal único utilizando Matlab. Métodos: seu desenvolvimento partiu da modelagem matemática do erro SPM, para ser codificado em Matlab eavaliar seu desempenho no canal para condições específicas. Para realizar as simulações se implementou um canal de comunicações em fibra óptica, tendo em conta os parâmetros que regem as redes XGPON ou 10GPON que são a seguinte geração de Rede Óptica Passiva com Capacidade de Gigabit (GPON). Para simular o canal de fibra óptica se definiramas distancias de 20,40 e $60 \mathrm{~km}$, eas potencias de 4, 10 e $20 \mathrm{~mW}$, com velocidades de transmissão de $10 \mathrm{Gbps}$, nas longitudes de onda de 1550 e $1310 \mathrm{~nm}$, usando a modulação QPSK. Os parâmetros para desenvolver a simulação consideraramas recomendações da União Internacional de Telecomunicações (ITU). Resultados: através de uma análise gráfica se identificaram os parâmetros que afetamo erro SPM, como a potência, a área efetiva, a distânciaeo tipo de fibra, entre outros. Conclusão: o erro SPM por sisónãoé prejudicial para as redes XGPON namodulação QPSK, considerando que a máxima diferença obtida no projeto foi de $28,8^{\circ}$, sempre e quando se tenham em conta a potência, a distância e os tipos de fibra, de acordo com as recomendações da ITU (G652, G987, G691 e G957).

Palavras-chave: Auto-modulação de fase, fibra óptica de canal único padrão, modulação por deslocamento de fasequaternário, rede óptica passiva com capacidade de gigabit. 


\section{Introducción}

Las actuales comunicaciones por medio de cables de fibra óptica ofrecen velocidades de trasmisión a los usuarios finales desde las unidades de Mbpshasta $2.5 \mathrm{Gbps}$, con distancias máximas de $60 \mathrm{~km}$ en la Red Óptica Pasiva con Capacidad de Gigabit (Gigabit Passive Optical Network, GPON).Según [1], [2], [3], este tipo de redes se han empezado a desplegar como solución de acceso rápido para el transporte de información.

El gran crecimiento y expansión de internet y servicios como televisión de alta definición, video llamadas, entre otros, exigen que aumente la necesidad de ancho de banda en las redes de telecomunicaciones actuales, lo que requiere una red de mayor eficiencia. De acuerdo con [4]-[6], es necesario realizar estudios minuciosos con el fin de mantener una alta calidad de transmisión de información, analizando los efectos que se producen al utilizar determinados parámetros en el diseño de las redes ópticas.

Por estas razones las redes de Fibra óptica Para El Hogar (Fiber To The Home, FTTH) basadas en el estándar de red GPON, deberán migrar a las redes XGPON o 10GPON, definidas por las recomendaciones G.987, G.987.1, G.987.2 y G.987.3, de la Unión Internacional de Telecomunicaciones (International Telecommunication Union, ITU), según [7], [8].

La información que transita por canales de fibra óptica sufre distorsiones debido a la aparición de efectos lineales y no lineales, los cuales restringen la velocidad de transmisión. La Automodulación de Fase (Self Phase Modulation, SPM) es uno de los principales errores no lineales presentes en los sistemas de comunicaciones, como lo indican [4], [6], [9]-[11],quienes evalúan el error SPM.

Este proyecto está enfocado a evaluar el desempeño de un canal de comunicaciones ópticas basado en el estándar XGPON con respecto a la incidencia del error SPM, utilizando el software Matlab como herramienta de simulación [12], y bajo condiciones específicas de funcionamiento establecidas de acuerdo con las normas.

\section{Materiales y métodos}

Para la implementación del canal de comunicación en fibra óptica, se identificó el modelo matemático que representa dicho canal en presencia del error SPM. La codificación del modelo en Matlab, se apoyó en la librería de código abierto Optilux [13], que se implementa como un conjunto de herramientas de Matlab y que proporciona las técnicas avanzadas para diseñar, simular y evaluar sistemas de comunicaciones ópticas.

La ecuación no lineal Schrödinger se usa para representar la propagación de los pulsos ópticos en las fibras ópticas, partiendo de esta ecuación se pueden modelar matemáticamente los errores que se presentan en las comunicaciones ópticas.

La ecuación no lineal de Schrödinger (NonLinear Schrödinger Equation, NLSE) en ausencia de efectos de polarización para un campo eléctrico en voltios/metro, siendo $z$ la distancia en metros y el tiempo en segundos, es(1), de acuerdo con los análisis[13], [14].

$$
\frac{\partial A(z, t)}{\partial z}=-\frac{\alpha}{2} A-\beta_{1} \frac{\partial A}{\partial t}+j \frac{\beta_{2}}{2} \frac{\partial^{2} A}{\partial t^{2}}+\frac{\beta_{3}}{6} \frac{\partial^{3} A}{\partial t^{3}}-j \gamma|A|^{2} A
$$

ISSN 0122-820X

ISSN 2422-5053

PP: $37-47$

Donde $j$ es la unidad imaginaria, $\beta_{k}=\left.\frac{d^{k}}{d w^{k}}\right|_{w=w_{0}}, \operatorname{con} k=1,2, \ldots$, siendo $\beta_{w}$ la constante de propagación, $w_{0}=2 \pi f_{0}=2 \pi c / \lambda_{0}$ la frecuencia central de $\boldsymbol{A}(z, t)$, la velocidad de la luz, el coeficiente no lineal, y la atenuación de la fibra; estos parámetros satisfacen las relaciones siguientes. 
No. 1

Enero - Junio 2017 ISSN 0122-820X E-ISSN 2422-5053 PP: $37-47$
La atenuación con unidades $\mathrm{m}^{-1}$ es una medida de la pérdida de potencia a lo largo de la distancia. Suponiendo todos los parámetros cero para analizar solo la atenuación de la onda, excluyendo a $\alpha$, la NLSE se vuelve (2).

$$
\frac{\partial A}{\partial z}=-\frac{\alpha}{2} A
$$

Cuya solución es $\boldsymbol{A}(z, t)=A(0, t) e^{-\frac{\alpha z}{2}}$.

La atenuación es generalmente expresada en $\mathrm{dB} / \mathrm{km}$. En un $\mathrm{dB}$ la escala de pérdida de potencia puede ser deliberada como (3).

$$
\begin{aligned}
& 10 \log _{10}\left(|A(z, t)|^{2}\right)-10 \log _{10}\left(|A(0, t)|^{2}\right) \\
= & \alpha z \cdot 10 \log _{10}
\end{aligned}
$$

Por lo tanto la relación entre la atenuación en y la atenuación en es según (4).

$\alpha\left[\mathrm{m}^{-1}\right]=\frac{\alpha[\mathrm{dB} / \mathrm{km}]}{10 \log _{10} e} \cdot 10^{-4}=10^{-4} \cdot \ln (10) \alpha[\mathrm{dB} / \mathrm{km}]$

La atenuación puede ser asociada con la longitud de atenuación $L_{A}=1 / \alpha$ como una medida de la distancia en donde el efecto de pérdida es significativo.

En el régimen no lineal de la NLSE, la ecuación se escribe como (5).

$$
\frac{\partial A}{\partial z}=-\frac{\alpha}{2} A-j \gamma|A|^{2} A
$$

Cuya solución es (6).

$A(z, t)=A(0, t) e^{\frac{-\alpha z}{2}-j \gamma|A(0 . t)|^{2} L_{e}}=A(0, t) e^{\frac{-\alpha z}{2}} e^{-j \Phi(\mathrm{z}, \mathrm{t})}$

De donde $\Phi(\mathrm{z}, \mathrm{t})$ es la rotación de fase SPM, mientras $L_{e}$ es la longitud efectiva. Para $\mathrm{z} \ll 1 / \alpha, L_{e}(\mathrm{z}) \cong \mathrm{z}$; mientras que para $\mathrm{z} \gg 1 / \alpha$, $\mathrm{L}_{\mathrm{e}}(\mathrm{z}) \cong \mathrm{L} \_\mathrm{A}$; por tal motivo, de acuerdo con Serena, Bertolini y Vannucci [12], resulta que es una medida de la distancia sobre la cual el efecto no lineal es significativo.

Las interacciones no lineales incrementan al aumentar la longitud de los enlaces de

fibras ópticas, las cuales van desapareciendo conforme se propagan las señales ópticas a través de la fibra, debido a la disminución de su potencia ocasionada por la atenuación. Por esta razón, los efectos no lineales se presentan en la primera etapa del trayecto de fibra, conocida como distancia efectiva, la cual está dada por (7).

$$
L_{e}=\frac{1-e^{-\alpha L}}{\alpha}
$$

Donde $\alpha$ es el coeficiente de atenuación. Al igual que la distancia, las interacciones no lineales se incrementan con la intensidad del campo incidente, el cual es inversamente proporcional al área transversal del núcleo de la fibra. Sin embargo, según [6], es más conveniente considerar el área transversal efectiva del modo de propagación fundamental, porque la distribución de la potencia no es totalmente uniforme con respecto al área transversal del núcleo.

Debido a las características de los componentes de la fibra óptica, su índice de refracción tiene un aporte lineal y un aporte no lineal, el cual está dado en (8).

$$
n=n_{0}+n_{2} \frac{P}{A_{e}}
$$

Donde es el índice de refracción no lineal que relaciona la dependencia que posee el índice de refracción con respecto a la intensidad de potencia $\mathrm{P}$ y el área efectiva de la fibra . Como consecuencia de esta dependencia, la contribución no lineal del índice de refracción da como resultado un cambio de fase de la señal óptica dado por (9).

$$
\Phi_{N L}=\gamma P L_{e}
$$

Donde es el coeficiente no lineal, la relación entre el coeficiente no lineal y el índice no lineal de la fibra se define mediante (10), según[6].

$$
\gamma=\frac{2 \pi n_{2}}{\lambda A_{e}}
$$


Para realizar las simulaciones se usó el entorno específico de las redes XGPON o 10GPON que son la siguiente generación de GPON. Debido al aumento de servicios se hace necesario un incremento en el ancho de banda. De acuerdo con [15], GPON posiblemente será reemplazado por la tecnología XGPON que proporciona un mayor ancho de banda, ofrece velocidades de transmisión de hasta 10Gbps de bajada y 2.5 Gbps de subida. El crecimiento evolutivo bajo XGPON se basa en la demanda de las inversiones mínimas de equipos. Así XGPON debe funcionar con la misma infraestructura que GPON, según[16]. Los operadores deben mantener la longitud de onda actual, de modo que haya una coexistencia con el actual GPON y las redes de acceso futuras. Sin embargo, en concordancia con [7], [8], [17], GPON actual y XGPON en convivencia, requieren la separación de longitud de onda de las señales para evitar interferencias entre los canales descendentes $\mathrm{y}$ ascendentes.

Siguiendo las recomendaciones de las normas para XGPON y las normas G.652, G.691 y G.957 de la ITU que definen las características físicas, las velocidades de trasmisión, las potencias y las longitudes de los cables de fibra óptica, como se representa en la Tabla I, se consultaron las especificaciones técnicas de los fabricantes reconocidos de fibra óptica que cumplieran con las anteriores recomendaciones, y con presencia en Latinoamérica.

Tabla I. Parámetros del canal

\begin{tabular}{|c|c|c|c|c|c|}
\hline \multirow{2}{*}{ Red } & $\begin{array}{c}\text { Velocidad de } \\
\text { transmisión en } \\
\text { Gbps }\end{array}$ & $\begin{array}{c}\text { Ventana de } \\
\text { transmisión en } \\
\mathbf{n m}\end{array}$ & Modulación & $\begin{array}{c}\text { Potencia en } \\
\mathbf{m W}\end{array}$ & $\begin{array}{c}\text { Distancia } \\
\text { en } \mathbf{K m}\end{array}$ \\
\hline \multirow{2}{*}{ XGPON } & 10 & 1550 & \multirow{2}{*}{ QPSK } & 4,10 y 20 & 20,40 y 60 \\
\cline { 2 - 3 } & 2.5 & 1310 & & 20 \\
\hline
\end{tabular}

Fuente: Autores

De acuerdo con lo anterior, se optó por la empresa Optral que fabrica y distribuye fibra óptica, quienes además de cumplir con las normas, proveen los parámetros necesarios para la simulación, como se ilustra en la Tabla II.

Tabla II.Propiedades ópticas de fibra monomodo estándar

\begin{tabular}{|c|c|c|c|}
\hline \multicolumn{2}{|c|}{ Propiedades ópticas } & G.652.B & G.652.D \\
\hline \multirow{2}{*}{ Diámetro del campo modal $(\mu \mathrm{m})$} & $1310 \mathrm{~nm}$ & $9.0 \pm 0.4$ & $9.0 \pm 0.4$ \\
\cline { 2 - 4 } & $1550 \mathrm{~nm}$ & $10.1 \pm 0.5$ & $10.1 \pm 0.5$ \\
\hline \multirow{4}{*}{\begin{tabular}{c} 
Coeficiente de atenuación $(\mathrm{dB} / \mathrm{km})$ \\
\cline { 2 - 4 }
\end{tabular}} & $1310 \mathrm{~nm}$ & $\leq 0.35$ & $\leq 0.35$ \\
\cline { 2 - 4 } & $1460 \mathrm{~nm}$ & $\leq 1.0$ & $\leq 0.35$ \\
\cline { 2 - 4 } & $1550 \mathrm{~nm}$ & --- & $\leq 0.21$ \\
\hline \multirow{2}{*}{$\begin{array}{c}\text { Dispersión cromática } \\
(\mathrm{ps} / \mathrm{nm} . \mathrm{km})\end{array}$} & $1625 \mathrm{~nm}$ & $\leq 0.23$ & $\leq 0.23$ \\
\cline { 2 - 4 } & $1285-1330 \mathrm{~nm}$ & $\leq 131$ & $\leq 131$ \\
\cline { 2 - 4 } & $1550 \mathrm{~nm}$ & $\leq 18$ & $\leq 18$ \\
\hline
\end{tabular}

Fuente: Autores

\section{Resultados y análisis}

A continuación se muestran los resultados de la simulación del efecto SPM en un pulso luminoso para un canal de comunicación de fibra óptica, utilizando el diagrama que representa la fase de la señal portadora en función del tiempo del símbolo y el diagrama de constelación; para estos diagramas se asignaron el color rojo para la señal en la fuente, y los colores verde, negro y azul para la señal obtenida a 20,40 
Automodulación de fase en redes ópticas pasivas de próxima generación, utilizando modulación por desplazamiento cuaternario de fase

y $60 \mathrm{~km}$, respectivamente. Para representar el comportamiento de la onda en el canal, se definieron las potencias de transmisión de 4, 10 y $20 \mathrm{~mW}$, considerando para cada caso 15 símbolos de la trama de bits enviados, longitud de onda $1550 \mathrm{~nm}$, velocidad de 10Gbps, longitudes de fibra óptica de 20, 40 y $60 \mathrm{~km}$, y la técnica de Modulación por Desplazamiento Cuaternario de Fase (Quaternary Phase Shift Keying, QPSK).
En la figura 1 se representa la fase de los pulsos luminosos en el canal de comunicación de fibra óptica para una potencia de transmisión de $4 \mathrm{~mW}$; en esta gráfica se identificó con ayuda de las herramientas del entorno de figura de Matlab, que la señal transmitida se desfasó $3.78^{\circ}, 5.22^{\circ}$ y $5.8^{\circ}$, para distancias del canal de 20, 40 y $60 \mathrm{~km}$, respectivamente.

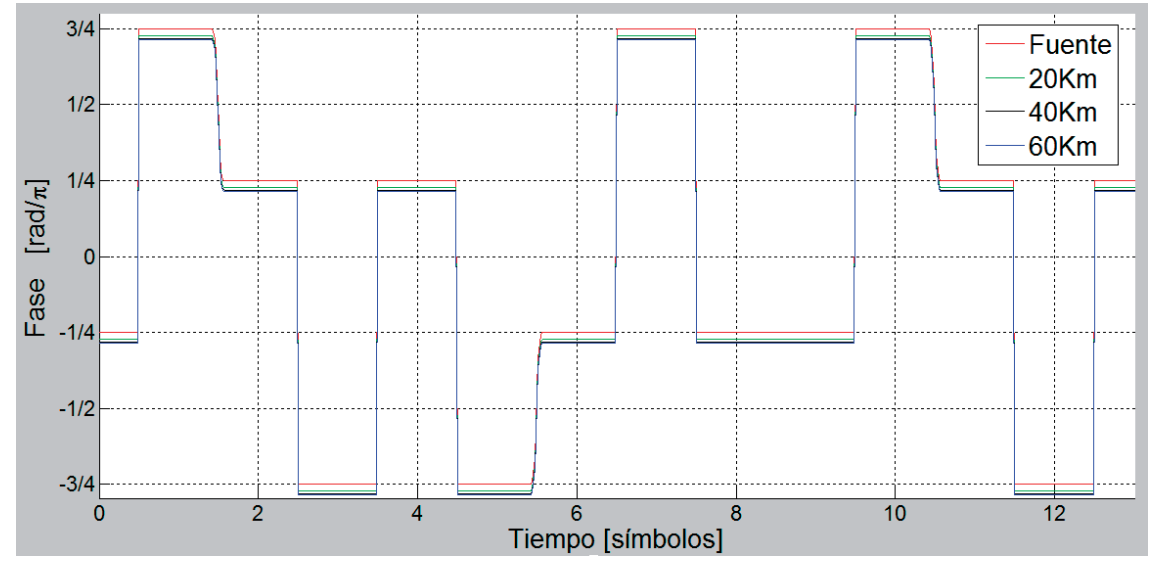

Figura. 1. Fase de la señal para potencia de $4 \mathrm{~mW}$. Fuente: [12]

En la figura 2 se muestra el diagrama de constelación para una potencia de $4 \mathrm{~mW}$, en donde el punto rojo representa la constelación en la fuente y los puntos de color verde, negro y azul, las constelaciones para distancias de fibra de 20, 40 y $60 \mathrm{~km}$, respectivamente. En esta figura se ilustra la atenuación de la señal a medida que aumenta la distancia, por ejemplo desde la fuente hasta una distancia de $60 \mathrm{~km}$, la señal se atenuó $3.84 \mathrm{~mW}$ aproximadamente, este valor se calculó a partir del dato obtenido con la herramienta data cursor en el diagrama de constelación.

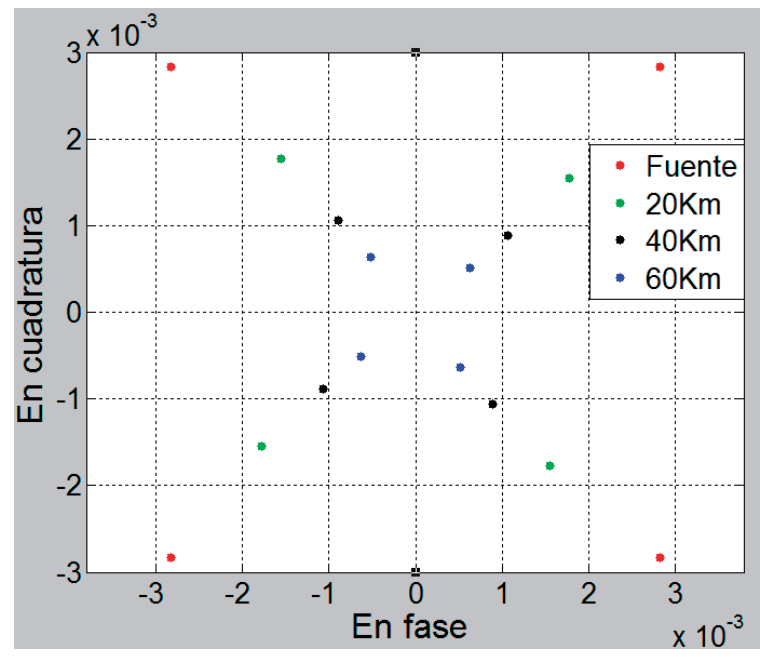

Figura 2. Diagrama de constelación para potencia de $4 \mathrm{~mW}$. Fuente: [12] 
En la figura 3 se representa la fase de los pulsos luminosos en el canal de comunicación de fibra óptica para una potencia de transmisión de $10 \mathrm{~mW}$, en ella, la señal transmitida se desfasó $11.52^{\circ}$, $13.86^{\circ}$ y $14.4^{\circ}$, para distancias del canal de 20,40 y $60 \mathrm{~km}$, respectivamente.

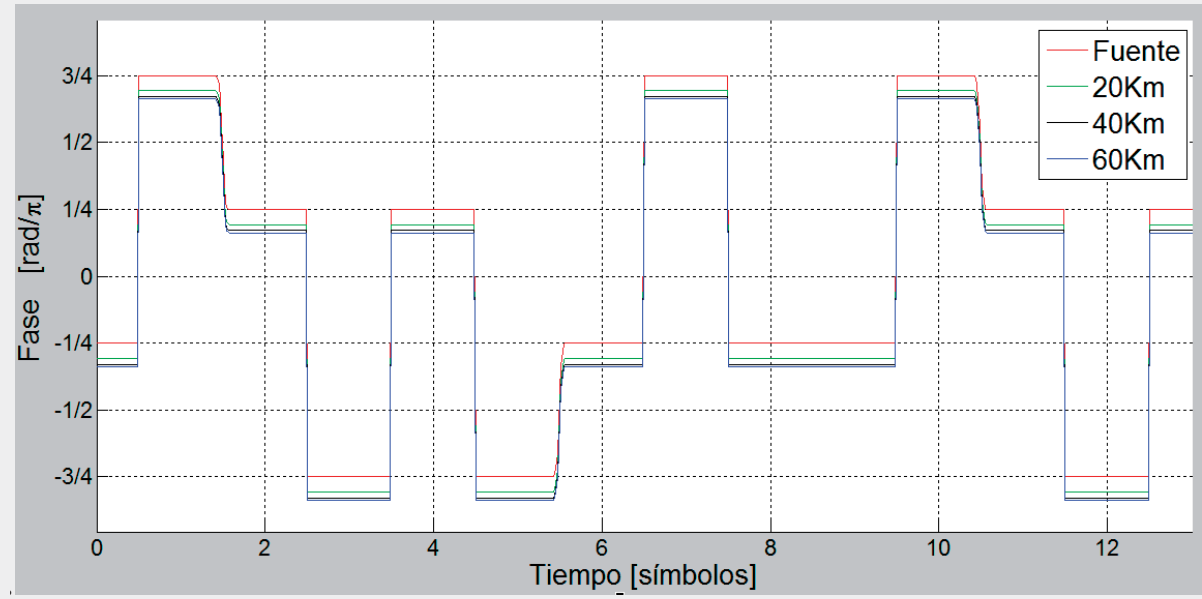

Figura 3. Fase de la señal para potencia de $10 \mathrm{~mW}$. Fuente: [12]

En la figura 4 se muestra el diagrama de constelación para una potencia de $10 \mathrm{~mW}$, en donde se ilustra la atenuación de la señal a medida que aumenta la distancia, por ejemplo desde la fuente hasta una distancia de $60 \mathrm{~km}$, la señal se atenuó $9.35 \mathrm{~mW}$ aproximadamente.

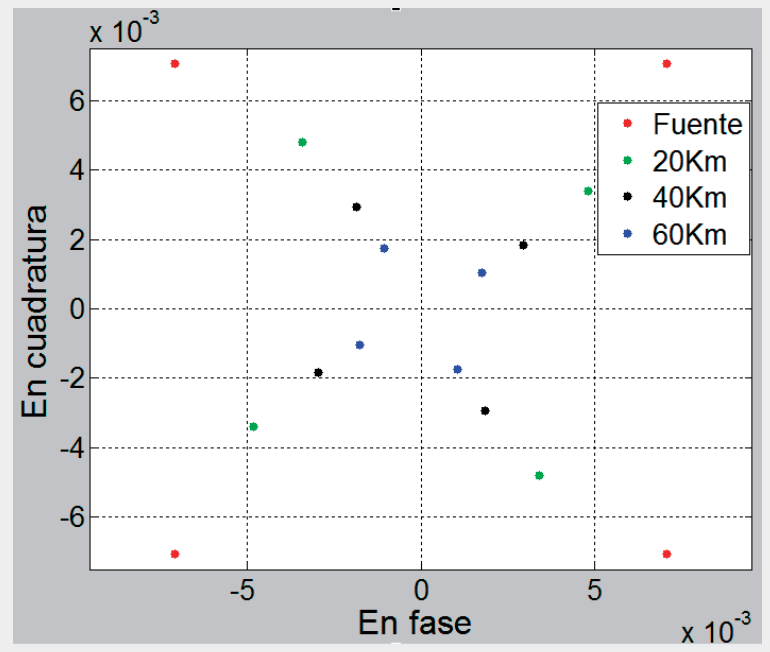

Figura 4. Diagrama de constelación para potencia de $10 \mathrm{~mW}$. Fuente: [12]

En la figura 5 se representa la fase de los pulsos luminosos en el canal de comunicación de fibra óptica para una potencia de transmisión de $20 \mathrm{~mW}$, ilustrando que la señal transmitida se desfasó $19.26^{\circ}, 25.92^{\circ}$ y $28.26^{\circ}$, para distancias del canal de 20,40 y $60 \mathrm{~km}$, respectivamente. Comparando los resultados de las potencias evaluadas, se infiere que el desfase de la señal aumenta de manera proporcional, conforme se incrementan la potencia de transmisión y la distancia de la fibra. 


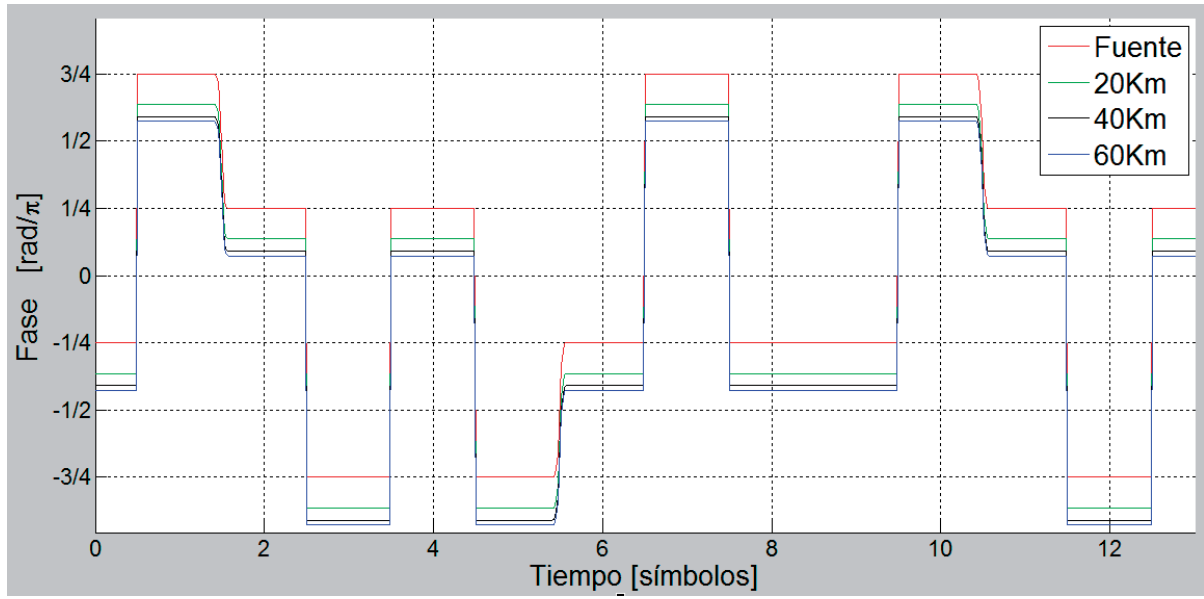

Figura 5. Fase de la señal para potencia de $20 \mathrm{~mW}$.

Fuente: [12]

En la figura 6 se ilustra la atenuación de la señal de $20 \mathrm{~mW}$ a medida que aumenta la distancia, por ejemplo desde la fuente hasta una distancia de $60 \mathrm{~km}$, la señal se atenuó $19.17 \mathrm{~mW}$ aproximadamente.

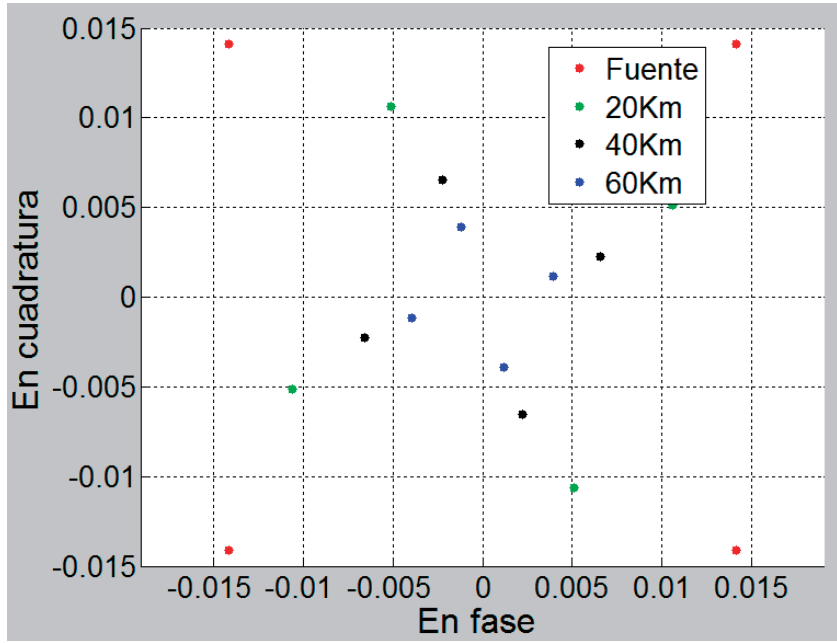

Figura 6. Diagrama de constelación para potencia de $20 \mathrm{~mW}$. Fuente: [12]

En las figura 7 y figura 8 se ilustra que el ángulo de desfase aumenta linealmente al incrementarse la potencia, y su pendiente está dada por .La separación entre las rectas es mayor en la figura 7 en comparación con la Figura 8; esto se debe a que la atenuación es mayor en la longitud de onda de $1310 \mathrm{~nm}$, por lo cual la longitud efectiva es más corta. Por ejemplo, la máxima longitud efectiva para $1550 \mathrm{~nm}$ y $1310 \mathrm{~nm}$ es de 18.9 y $12.5 \mathrm{~km}$ respectivamente, teniendo en cuenta a (7) y los datos de la Tabla II.

Aunque la longitud efectiva es más corta, el desfase es mayor en la longitud de $1310 \mathrm{~nm}$, esto se debe a que el área efectiva es menor en la longitud de onda de 1550nm. 


\section{Yhon Edinson Estevez-Mendoza, Byron Medina-Delgado, Luis Leonardo Camargo-Ariza}

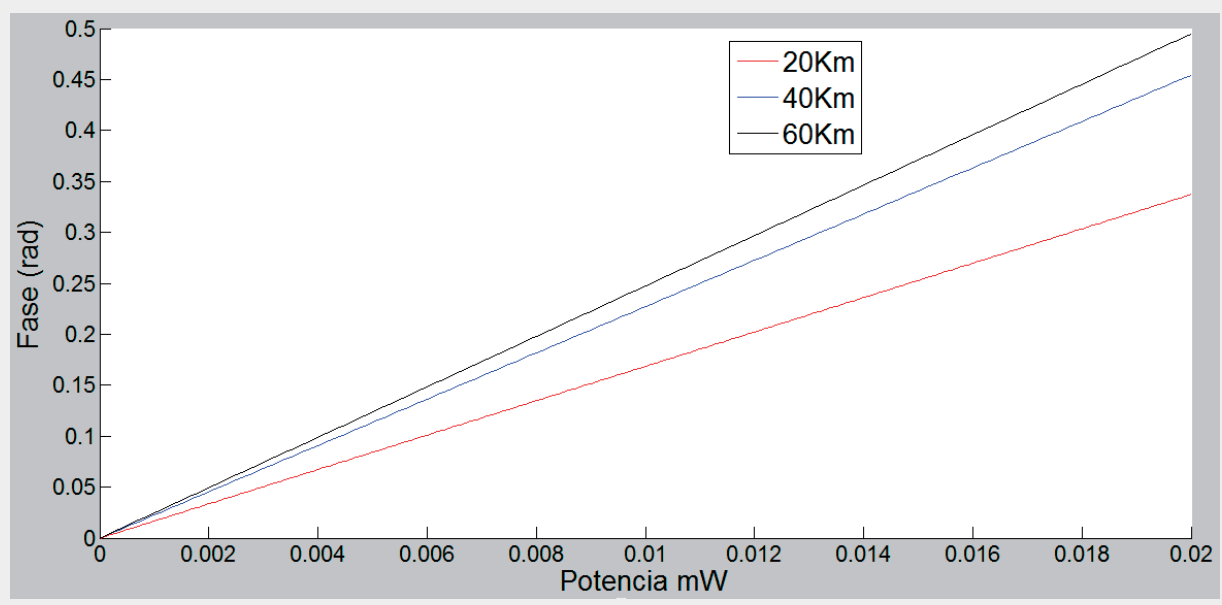

Figura 7. Desfase para una longitud de onda de $1550 \mathrm{~nm}$. Fuente: [12]

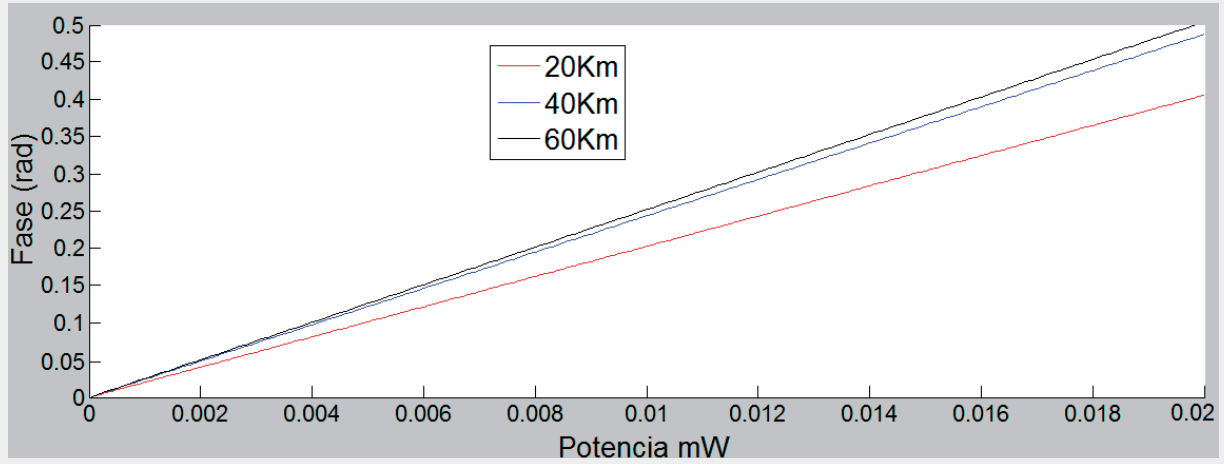

Figura 8. Desfase para una longitud de onda de $1310 \mathrm{~nm}$. Fuente: [12]

Para identificar la influencia del área efectiva en el SPM, se varió el área desde 50hasta 150 para longitudes de onda de 1310 y $1550 \mathrm{~nm}$, con potencia de $20 \mathrm{~mW}$ y longitud de la fibra de $60 \mathrm{~km}$, considerando las normas y las especificaciones de la Tabla II. En la Figura 9 se aprecia que el desfase se reduce a medida que el área efectiva se incrementa.

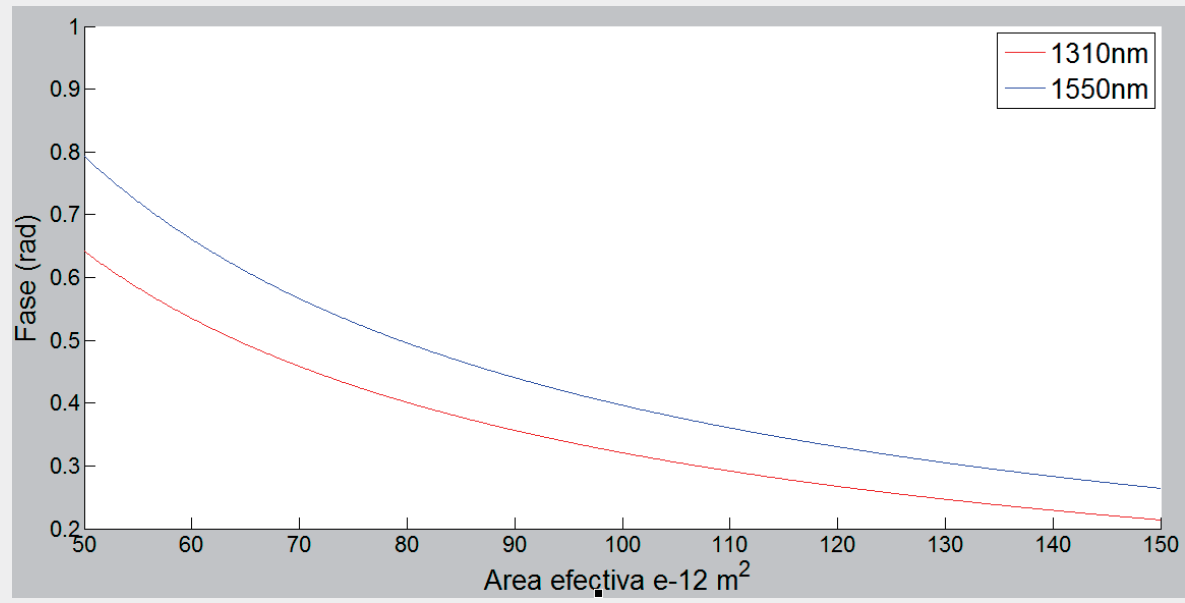

Figura 9. Desfase en función del área efectiva. Fuente: [12] 
No. 1

Enero - Junio 2017 ISSN 0122-820X E-ISSN 2422-5053 PP: $37-47$
Al evaluar la probabilidad de error de bit se encontró que el sistema simulado con la técnica de modulación QPSK no produce errores, debido a que el desfase de la señal no superó los $45^{\circ}$ necesarios para producir errores de bit; como se ilustra en las figura 1 a figura6, el máximo desfase obtenido en las simulaciones fue de $28.8^{\circ}$.

\section{Conclusiones}

El error SPM por sí solo no es perjudicial para las redes XGPON en la modulación QPSK, considerando que el máximo desfase obtenido en el proyecto fue de $28.8^{\circ}$; siempre y cuando se tengan en cuenta la potencia, la distancia y los tipos de fibra de acuerdo con las recomendaciones de la ITU (G652, G987, G691 y G957). Este desfase podría ser perjudicial para modulaciones en las que el número de estados de fase es mayor a 4, debido a que el margen de desfase entre los estados es menor.

Una de las variables que produce el SPM es la potencia incidente. Cuando se tiene un cable de fibra óptica de longitud determinada, el desfase producido por el SPM aumenta linealmente conforme aumenta la potencia, con una pendiente proporcional al producto del coeficiente no lineal y la longitud efectiva. Esto quiere decir que si se mantiene la distancia y el tipo de fibra, se puede predecir linealmente el desfase al variar la potencia.

El error SPM es dependiente del área efectiva de la fibra óptica y disminuye a medida que esta área aumenta. Al variar el área efectiva de 50 a 100 , el desfase disminuye de $0.2 \pi$ a $0.1 \pi$ y de $0.25 \pi$ a $0.126 \pi$, para longitudes de onda de $1310 \mathrm{~nm}$ y $1550 \mathrm{~nm}$, respectivamente; es decir,una disminución aproximada del $50 \%$ del error. Además, si el área efectiva se incrementa a 150 , el desfase disminuye de $0.2 \pi$ a $0.06 \pi$ y $0.25 \pi$ a $0.084 \pi$, para longitudes de onda de $1310 \mathrm{~nm}$ y $1550 \mathrm{~nm}$, respectivamente; es decir, una disminución aproximada del $67 \%$ del error. Esto evidencia que el error SPM depende del área efectiva; además, se puede evaluar el tamaño de dicha área que no afecta a otros errores en el canal.

La simulación de un sistema de comunicación de fibra óptica a través de herramientas computacionales, exige la caracterización de los componentes del sistema a través de la especificación de los atributos de atenuación, velocidad de trasmisión, distancia del enlace, potencia de transmisión, y tipos de fibra óptica, entre otros; estos datos deben obtenerse de las especificaciones de los fabricantes, y deben cumplir con las recomendaciones de la ITU; con el propósito de alcanzar la mejor aproximación del escenario real.

Herramientas de evaluación del canal como el diagrama de ojo y el error de bit no aplican para observar los efectos producidos en la señal debido al error SPM, porque no permiten comparar la fase de la señal en la fuente con la recibida.

\section{Referencias}

[1] C. Hernández, V. Gutierrez y D. Espinosa, "Impacto y masificación del uso de las redes GPON en Colombia frente a otras tecnologías",Redes Ing, vol. 2, no. 1, pp. 86-99, May 2011.

[2] C. Serpa y A. Correa, "Análisis de la expansión de redes de acceso pasivas de fibra óptica GPON y BPON en la ciudad de Medellín", Rev. Tecnologías, no. 24, pp. 59-70, Jun. 2010.

[3] J.J. Granada-Torres, A.M. CárdenasSoto, N. Guerrero-González, "Redes ópticas elásticas: un nuevo paradigma en las futuras redes de telecomunicaciones", Respuestas, vol. 20, no. 2, pp. 6-22, 2015.

[4] M. Jimenez y C. Carrera, "Estudio $\mathrm{y}$ simulación del efecto no lineal 
automodulación de fase", Revista politécnica, vol 35, no 1, 2015.

[5] J. Álvarez, "Ecualización de canales ópticos de comunicación utilizando técnicas de procesamiento digital de señales sobre sistemas ópticos basados en el estándar GPON", proyecto de investigación GIDT, Departamento de electricidad y electrónica, Universidad Francisco de Paula Santander, San José de Cúcuta, 2011.

[6] A. Gil, A. Cárdenas and C. Carmona, "Benefits and drawbacks of SPM in single- channel $10 \mathrm{gb} / \mathrm{s}$ QPSK transmission",IEEE, no. 53, pp. 5, 2012.

[7] J. Pinto, "Evolution of FTTH Networks for NG-PON2",PT Inovação e Sist, pp. 4-11, 2013.

[8] V. Bostjan, M. Jurij, L. Vitalii and Y. Ignatov, "Optical Access Network Migration from GPON to XG-PON",in The third international conference on access networks, pp. 62-67, 2012.

[9] H. Castellanos y C. Collazos, "Estudio de los efectos de automodulación de fase (SPM) en fibras ópticas para sistemas DWDM, Banda C (ITU)",Rev. Colomb. Física, vol. 44, no. 2, pp. 176-184, Aug. 2012.

[10] A.G. García, "Análisis de Atenuación, Dispersión y Automodulación de Fase en Sistemas de Comunicaciones de Único Canal por Fibra Óptica a $10 \mathrm{~Gb} /$ s", Centro de Investigaciones en Optica, AC, Guanajuato, México, 2007.

[11] J.P. Agredo, J.G. López, A. Toledo y H. Ordóñez, "Efectos no lineales y su relación con los parámetros de transmisión de una red WDM",Rev. Fac. Ing,vol. 20, no. 31, pp. 23-35, Jul. 2011.
[12] MathWorks Inc. "Matlab The language technical Computing", Noviembre 2013. [Online]. Available: http://www. mathworks.com/products/matlab/.

[13] P.Serena, M. Bertolini, andA. Vannucci, “Optilux Toolbox",Departament of information engineering, University of Palma, Italy, March, 2009.

[14] G. Agrawal, "Nonlinear Fiber Optics", Academic Press, p. 467, 2001.

[15] M. Abdalla, S. Idrus and B. Mohammad, "Hybrid TDM-WDM 10G-PON for high scalability next generation PON",in Proc. IEEE 8th Conf. Ind. Electron. Appl. ICIEA, pp. 1448-1450, 2013.

[16] J. Mullerova, D. Korcek and M. Dado, "On wavelength blocking for XGPON coexistence with GPON and WDM-PON networks," in Int. Conf. Transparent Opt. Networks, pp. 5-8, 2012.

[17] G. Kramer, M. De Andrade, R. Roy and P. Chowdhury, "Evolution of optical access networks: Architectures and capacity upgrades",in Proc. of IEEE, vol. 100, no. 5, pp. 1188-1196, May 2012.
Enero - Junio 2017 ISSN 0122-820X

E-ISSN 2422-5053 PP: $37-47$ 